Zbigniew Grande*, Institute of Mathematics, Pedagogical University, Plac

Weyssenhoffa 11, 85-072 Bydgoszcz, Poland. e-mail:

grande@wsp. bydgoszcz.pl

\title{
ON A.C. LIMITS AND MONOTONE LIMITS OF SEQUENCES OF JUMP FUNCTIONS
}

\begin{abstract}
The a.c. limits (introduced by Császár and Laczkovich) and the monotone limits of sequences of functions having everywhere finite unilateral limits are investigated.
\end{abstract}

Let $\mathbb{R}$ be the set of all reals and let $\mathcal{A}$ be a family of functions from $\mathbb{R}$ to $\mathbb{R}$. A function $f: \mathbb{R} \rightarrow \mathbb{R}$ belongs to the class $B_{1}^{*}(\mathcal{A})$ if there is a sequence of functions $f_{n} \in \mathcal{A}$ with $f=$ a.c. $\lim _{n \rightarrow \infty} f_{n}$, i.e. for each point $x \in \mathbb{R}$ there is a positive integer $k$ such that $f_{n}(x)=f(x)$ for every $n>k$.

It is evident that $f \in B_{1}^{*}(\mathcal{A})$ if and only if there is a sequence of sets $A_{n}$, $n=1,2, \ldots$, such that for each positive integer $n$ there is a function $g_{n} \in \mathcal{A}$ such that

$$
g_{n} / A_{n}=f / A_{n}, \quad A_{1} \subset A_{2} \subset \ldots \subset A_{n} \ldots
$$

and

$$
\mathbb{R}=\bigcup_{n=1}^{\infty} A_{n}
$$

In $[2,3]$ it is proved that in the case of the class $\mathcal{C}$ of all continuous functions the sets $A_{n}, n=1,2, \ldots$, can be closed and that $f \in B_{1}^{*}(\mathcal{C})$ if and only if for every nonempty closed set $A \subset \mathbb{R}$ there is an open interval $I$ such that $I \cap A \neq \emptyset$ and the reduced function $f /(A \cap I)$ is continuous.

In this article we will investigate the family $B_{1}(\mathcal{A})$, where $\mathcal{A}$ is the class $\mathcal{P}$ of all functions $f: \mathbb{R} \rightarrow \mathbb{R}$ such that for each point $x \in \mathbb{R}$ there are the both finite unilateral limits $\lim _{t \rightarrow x-} f(t)$ and $\lim _{t \rightarrow x+} f(t)$. The pointwise limits of sequences of such functions from $\mathcal{P}$ were investigated in [4]. Moreover it is well known ([5], p. 45) that if $f \in \mathcal{P}$ then the set $D(f)$ of all discontinuity points of $f$ is countable.

\footnotetext{
Key Words: upper semicontinuity, decreasing sequences of functions, $B_{1}^{*}$ class, a.c. convergence, jump function

Mathematical Reviews subject classification: 26A15, 26A21, 26A99

Received by the editors September 7, 1998

* Supported by Bydgoszcz Pedagogical University grant 1998
} 


\section{The A.C. Convergence}

Theorem 1. If $f \in B_{1}^{*}(\mathcal{P})$ then $f$ satisfies the following condition

(T) there is a countable set $A \subset \mathbb{R}$ such that the reduced function $f /(\mathbb{R} \backslash A) \in$ $B_{1}^{*}(\mathcal{C}(\mathbb{R} \backslash A))$, where $\mathcal{C}(\mathbb{R} \backslash A)$ denotes the class of all continuous functions $g: \mathbb{R} \backslash A \rightarrow \mathbb{R}$.

Proof. Since $f \in B_{1}^{*}(\mathcal{P})$, there is a sequence of functions $f_{n} \in \mathcal{P}, n=1,2 \ldots$, such that $f=$ a.c. $\lim _{n \rightarrow \infty} f_{n}$. Put

$$
A=\bigcup_{n=1}^{\infty} D\left(f_{n}\right)
$$

and observe that the set $A$ is countable. Since the functions $g_{n}=f_{n} /(\mathbb{R} \backslash A)$ are continuous for $n=1,2, \ldots$ and $f /(\mathbb{R} \backslash A)=$ a.c. $\lim _{n \rightarrow \infty} g_{n}$, the proof is completed.

The following Example shows that the condition $(\mathrm{T})$ is not sufficient for the relation $f \in B_{1}^{*}(\mathcal{P})$.

Example 1. Let

$$
g(x)=\sin \frac{1}{x} \text { for } x \neq 0, g(0)=1
$$

and

$$
f(x)=\sum_{n=1}^{\infty} \frac{g\left(x-w_{n}\right)}{2^{n}},
$$

where $\left(w_{n}\right)_{n}$ is an enumeration of all rationals such that $w_{n} \neq w_{m}$ for $n \neq m$, $n, m=1,2, \ldots$.

As the sum of an uniformly converging series of functions which are continuous at each irrational point, the function $f$ is continuous at every irrational point. Denote by $A$ the set of all rationals and observe that the reduced function $f /(\mathbb{R} \backslash A)$ is continuous. So the function $f$ satisfies the condition (T).

Now we will prove that $f$ is not in the class $B_{1}^{*}(\mathcal{P})$. Assume, by way of contradiction, that there is a sequence of functions $f_{n} \in \mathcal{P}, n=1,2, \ldots$, such that a.c. $\lim _{n \rightarrow \infty} f_{n}=f$. Then there is a positive integer $k$ and a set, $B$, of the second category such that $f_{m}(x)=f(x)$ for each point $x \in B$ and $m \geq k$. Consequently, the set

$$
E=\left\{x ; f_{m}(x)=f(x) \text { for } m \geq k\right\} \supset B
$$


is Borelian and of the second category. There is an open interval $I=(a, b)$ such that the set $I \backslash E$ is of the first category. Let $u \in I$ be a rational point such that $u=w_{i}$ for some positive integer $i>k$. Since all functions

$$
x \rightarrow g\left(x-w_{n}\right), \quad n \neq i, \quad n=1,2, \ldots,
$$

are continuous at the point $u$, the function

$$
h(x)=\sum_{i \neq n=1}^{\infty} \frac{g\left(x-w_{n}\right)}{2^{n}}
$$

is also continuous at $u$. Let $J \subset I$ be an open interval containing $u$ such that

$$
|h(t)-h(u)|<\frac{1}{8^{i}} \text { for } t \in J .
$$

There are sequences of points

$$
u<u_{j}, v_{j} \in J \cap E, \quad j=1,2, \ldots
$$

such that

$$
\begin{gathered}
u=\lim _{j \rightarrow \infty} u_{j}=\lim _{j \rightarrow \infty} v_{j} \\
\left|g\left(u_{j}-u\right)\right|<\frac{1}{8^{i}} \text { and }\left|g\left(v_{j}-u\right)\right|>1-\frac{1}{8^{i}} \text { for } j=1,2, \ldots
\end{gathered}
$$

Observe that

$$
f_{i}\left(u_{j}\right)=f\left(u_{j}\right)=h\left(u_{j}\right)+\frac{g\left(u_{j}-w_{i}\right)}{2^{i}}<h(u)+\frac{1}{8^{i}}+\frac{1}{8^{i} 2^{i}}=h(u)+\frac{2^{i}+1}{2^{i} 8^{i}},
$$

and

$$
\begin{gathered}
f_{i}\left(v_{j}\right)=f\left(v_{j}\right)=h\left(v_{j}\right)+\frac{g\left(v_{j}-w_{i}\right)}{2^{i}}>h(u)-\frac{1}{8^{i}}+\frac{1}{2^{i}}-\frac{1}{2^{i} 8^{i}}= \\
h(u)+\frac{1}{2^{i}}-\frac{2^{i}+1}{8^{i} 2^{i}}=h(u)+\frac{8^{i}-2^{i}-1}{8^{i} 2^{i}}>h(u)+\frac{\left(2^{i}+2\right)}{8^{i} 2^{i}},
\end{gathered}
$$

a contradiction with the assumption that $f_{i}$ has the finite limit from the right hand side. So $f$ is not in $B_{1}^{*}(\mathcal{P})$.

Theorem 2. Let $f$ be a function such that there is a countable set $A$ and a $G_{\delta}$-set $B \subset A$ for which the reduced function $f /(\mathbb{R} \backslash A)$ is continuous and for each point $x \in A \backslash B$ there are the finite unilateral limits

$$
\lim _{\mathbb{R} \backslash A \ni t \rightarrow x-} f(t) \text { and } \lim _{\mathbb{R} \backslash A \ni t \rightarrow x+} f(t) .
$$

Then the function $f \in B_{1}^{*}(\mathcal{P})$. 
Proof. Let $\left(U_{n}\right)_{n}$ be a sequence of open sets such that

$$
B=\bigcap_{n=1}^{\infty} U_{n} \text { and } U_{1} \supset \ldots \supset U_{n} \supset \ldots
$$

Since every function $f$ with the finite set of all discontinuity points belongs to $B_{1}^{*}(\mathcal{P})$, without loss of the generality we can assume that the set $A$ is infinite. Enumerate all points of the set $A$ in a sequence $\left(a_{n}\right)_{n}$ such that $a_{n} \neq a_{m}$ for $n \neq m, n, m=1,2, \ldots$

Next fix a positive integer $n$ and put

$$
f_{n}(x)=f(x) \text { for } x \in\left(\mathbb{R} \backslash U_{n}\right) \backslash A \text {. }
$$

For $i \leq n$ we define also

$$
f_{n}\left(a_{i}\right)=f\left(a_{i}\right)
$$

and for other $x \in A \backslash U_{n}$ (i.e. $x=a_{i}$, where $i>n$ ) let either

$$
f_{n}(x)=\lim _{\mathbb{R} \backslash A \ni t \rightarrow x+} f(t)
$$

if $x$ is the left endpoint of a component of the set $U_{n}$ or

$$
f_{n}(x)=\lim _{\mathbb{R} \backslash A \ni t \rightarrow x-} f(t)
$$

otherwise.

Finishing we define $f_{n}$ as the linear function on the closures of all components of the set $U_{n} \backslash\left\{a_{i} ; i \leq n\right\}$. Then

$$
f_{n} \in \mathcal{P} \text { for } n=1,2, \ldots \text { and } f=\text { a.c. } \lim _{n \rightarrow \infty} f_{n}
$$

so $f \in B_{1}^{*}(\mathcal{P})$ and the proof is completed.

For the formulation of the generalization of the last theorem we introduce the following notion:

A set $A$ is said to be an interval set if there is a sequence of nondegenerate intervals $I_{n}, n=1,2, \ldots$, such that

$$
A=\bigcup_{n=1}^{\infty} I_{n}
$$


Theorem 3. Let $f$ be a function such that there is a countable set $A$ such that the reduced function $f /(\mathbb{R} \backslash A)$ is continuous. Moreover, suppose that there is a sequence of interval sets $A_{n}, n=1,2, \ldots$, such that

$$
B=\bigcap_{n=1}^{\infty} A_{n} \subset A \text { and } A_{1} \supset \ldots A_{n} \supset \ldots
$$

and every reduced function $f /\left(\mathbb{R} \backslash A_{n}\right), n=1,2, \ldots$, has the finite limit at every point $x$ such that $x$ is an endpoint of a component of the set $A_{n}$ but $x$ does not belong to $A_{n}$. Then $f \in B_{1}^{*}(\mathcal{P})$.

Proof. The proof is similar as the proof of Theorem 2.

The following example shows that the assumption of Theorem 3 is essentially more general than that in Theorem 2 .

Example 2. Let $g$ be the same function as that from Example 1 and let $C \subset[0,1]$ be the ternary Cantor set. Put

$$
[0,1] \backslash C=\bigcup_{n=1}^{\infty}\left(a_{n}, b_{n}\right),
$$

where $\left(a_{n}, b_{n}\right), n=1,2, \ldots$, are components of the open set $[0,1] \backslash C$. For each positive integer $n$ we find a point

$$
c_{n} \in\left(a_{n}, b_{n}\right) \text { such that } g\left(c_{n}-a_{n}\right)=0 .
$$

Let

$$
f(x)=\frac{g\left(x-a_{n}\right)}{n} \text { for } x \in\left[a_{n}, c_{n}\right], \quad n=1,2, \ldots
$$

and

$$
f(x)=0 \text { otherwise on } \mathbb{R} .
$$

Observe that for every countable set $A$ the reduced function $f /(\mathbb{R} \backslash A)$ does not have the limit from the right hand side for any point $a_{n}, n=1,2, \ldots$ Evidently, there is not a countable $G_{\delta}$-set containing the set $B=\left\{a_{n} ; n=\right.$ $1,2, \ldots\}$. But if $A=B$ and for $n=1,2, \ldots$ we define

$$
A_{n}=\bigcup_{k=1}^{\infty}\left[a_{k}, \min \left(a_{k}+\frac{1}{n}, c_{k}\right)\right)
$$

then the hypothesis of Theorem 3 is satisfied and the function $f \in B_{1}^{*}(\mathcal{P})$. 
Next example shows that the assumption of Theorem 3 is not a necessary condition for the relation $f \in B_{1}^{*}(\mathcal{P})$.

Example 3. Let $C$ and $\left(a_{n}, b_{n}\right), n=1,2, \ldots$, be the same as those in Example 2. Find a countable set $B \subset C \backslash\left\{a_{n}, b_{n} ; n=1,2, \ldots\right\}$ which is dense in $C$. Enumerate all points of the set $B$ in a sequence $\left(z_{n}\right)_{n}$ such that $z_{n} \neq z_{m}$ for $n \neq m, n, m=1,2, \ldots$. For every positive integer $n$ find a sequence of closed intervals $I_{n, m}=\left[c_{n, m}, d_{n, m}\right] \subset(0,1), m=1,2, \ldots$, such that:

$$
\begin{aligned}
& \text { if }(n, m) \neq(k, l) \text { then } I_{n, m} \cap I_{k, l}=\emptyset, k, l, m, n=1,2, \ldots ; \\
& I_{n, m} \cap C=\emptyset \text { for } n, m=1,2, \ldots ; \\
& \lim _{m \rightarrow \infty} c_{n, m}=\lim _{m \rightarrow \infty} d_{n, m}=z_{n} \text { for } n=1,2, \ldots .
\end{aligned}
$$

For all positive integers $n, m$ define a continuous function

$$
f_{n, m}: I_{n, m} \rightarrow\left[0, \frac{1}{n}\right]
$$

such that

$$
f_{n, m}\left(c_{n, m}\right)=f_{n, m}\left(d_{n, m}\right)=0 \text { and } f_{n, m}\left(I_{n, m}\right)=\left[0, \frac{1}{n}\right] .
$$

Let

$$
f(x)=\left\{\begin{array}{ccl}
f_{n, m}(x) & \text { if } & x \in I_{n, m}, n, m=1,2, \ldots \\
n^{-1} & \text { if } & x=z_{n}, n=1,2, \ldots \\
0 & \text { otherwise on } & \mathbb{R} .
\end{array}\right.
$$

Observe that for any countable set $A$ there is a point in $B$ where the unilateral limit of the reduced function $f /(\mathbb{R} \backslash A)$ does not exist. Since every interval set containing $B$ is residual in the set $C$, the function $f$ does not satisfy the assumption of Theorem 3 .

But we will prove that $f \in B_{1}^{*}(\mathcal{P})$. For this for every positive integer $n$ define

$$
f_{n}(x)=\left\{\begin{array}{ccl}
f_{i, k}(x) & \text { for } & x \in I_{i, k}, i, k \leq n \\
i^{-1} & \text { for } & x=z_{i}, i \leq n \\
0 & \text { otherwise on } & \mathbb{R}
\end{array}\right.
$$

and observe that

$$
f_{n} \in \mathcal{P} \text {, and a.c. } \lim _{n \rightarrow \infty} f_{n}=f .
$$

Theorem 4. A function $f$ is the a.c. limit of a sequence of functions $f_{n} \in \mathcal{P}$ if and only if it satisfies the following condition: 
(P) there is a countable set $A$, a sequence of closed sets $A_{n}$ and a sequence of functions $g_{n} \in \mathcal{P}, n=1,2, \ldots$, such that

$$
\mathbb{R}=\bigcup_{n=1}^{\infty} A_{n} \text { and } A_{n} \subset A_{n+1} \text { for } n=1,2, \ldots
$$

and

$$
g_{n} /\left(A_{n} \backslash A\right)=f /\left(A_{n} \backslash A\right) \text { for } n=1,2, \ldots
$$

Proof. Sufficiency. For the proof of the sufficiency of condition $(\mathrm{P})$ we enumerate the set $A$ in a sequence $\left(a_{n}\right)_{n}$ and for $n=1,2, \ldots$ we define

$$
f_{n}(x)=\left\{\begin{array}{ccl}
f\left(a_{i}\right) & \text { for } & i \leq n \\
g_{n}(x) & \text { otherwise on } & \mathbb{R} .
\end{array}\right.
$$

Then

$$
\text { a.c. } \lim _{n \rightarrow \infty} f_{n}=f \text { and } f_{n} \in \mathcal{P} \text { for } n=1,2, \ldots
$$

Necessity. Let

$$
A=\bigcup_{n=1}^{\infty} D\left(f_{n}\right)
$$

where $D\left(f_{n}\right)$ denotes the set of all discontinuity points of the function $f_{n}$, $n=1,2, \ldots$.

Now, we will apply the transfinite induction.

Since $f=a . c . \lim _{n \rightarrow \infty} f_{n}$, there is a positive integer $k_{0}$ such that the set

$$
B_{k_{0}}=\left\{x: \forall_{i \geq k_{0}} f_{i}(x)=f(x)\right\}
$$

is of the second category. Consequently, there is an open interval $I_{0}$ with rational endpoints such that

$$
I_{0} \cap B_{k_{0}} \neq \emptyset \text { and } f_{i}(x)=f(x) \text { for all } x \in I_{0} \backslash A \text { and } i \geq k_{0} .
$$

Fix an ordinal number $\alpha>0$ and suppose that for every ordinal number $\beta<\alpha$ there are a positive integer $k_{\beta}$ and an open interval $I_{\beta}$ with rational endpoints such that

$$
\begin{gathered}
E_{\beta}=\left(I_{\beta} \backslash A\right) \backslash \bigcup_{\gamma<\beta} I_{\gamma} \neq \emptyset, \\
f_{i}(x)=f(x) \text { for } x \in E_{\beta} \text { and } i \geq k_{\beta} .
\end{gathered}
$$

and

$$
D_{\alpha}=\mathbb{R} \backslash \bigcup_{\beta<\alpha} I_{\beta} \neq \emptyset
$$


For each point $x \in D_{\alpha}$ there is a positive integer $k(x)$ such that

$$
f_{i}(x)=f(x) \text { for } i \geq k(x) .
$$

By Baire's category theorem there is a positive integer $k_{\alpha}$ such that the set

$$
F_{\alpha}=\left\{x \in D_{\alpha} ; k(x)=k_{\alpha}\right\}
$$

is of the second category in $D_{\alpha}$. So, there is an open interval $I_{\alpha}$ with rational endpoints such that

$$
D_{\alpha} \cap I_{\alpha} \neq \emptyset
$$

and

$$
f_{i}(x)=f(x) \text { for } x \in E_{\alpha} \text { and } i \geq k_{\alpha} .
$$

Let $\alpha_{0}$ be the first ordinal number $\alpha$ with $E_{\alpha}=\emptyset$. Since the family of all intervals with rational endpoints is countable, $\alpha_{0}$ is a countable ordinal number. Every set $I_{\alpha} \cap D_{\alpha}, \alpha<\alpha_{0}$, is an $F_{\sigma}$ set, so

$$
I_{\alpha} \cap D_{\alpha}=\bigcup_{n=1}^{\infty} F_{n, \alpha},
$$

where all sets $F_{n, \alpha}, n=1,2, \ldots, \alpha<\alpha_{0}$, are closed. Enumerate the set $A$ in a sequence $\left(a_{n}\right)_{n}$ and all sets $F_{n, \alpha}, n=1,2, \ldots, \alpha<\alpha_{0}$, in a sequence $\left(F_{k_{i}, \alpha_{i}}\right)_{i}$. For $n=1,2, \ldots$ let

$$
A_{n}=\bigcup_{i=1}^{n} F_{k_{i}, \alpha_{i}}
$$

and

$$
g_{n}=\left\{\begin{array}{ccc}
f\left(a_{i}\right) & \text { for } & i \leq n \\
f_{\max \left(k_{\alpha_{1}}, \ldots, k_{\alpha_{n}}\right)} & \text { otherwise on } & \mathbb{R} .
\end{array}\right.
$$

Then

$$
g_{n} /\left(A_{n} \backslash A\right)=f /\left(A_{n} \backslash A\right)
$$

and the functions $g_{n} \in \mathcal{P}$ for $n=1,2, \ldots$ and

$$
f=\text { a.c. } \lim _{n \rightarrow \infty} f_{n} \text {. }
$$

So the proof is completed. 


\section{Monotone Convergence}

Remark 1. It is obvious that a function $f$ is the limit of a pointwise converging sequence of functions $f_{n} \in \mathcal{P}$ if and only if there is a Baire 1 function $g$ and a countable set $A$ such that

$$
\{x: f(x) \neq g(x)\} \subset A\} .
$$

Proof. Necessity. If

$$
f_{n} \in \mathcal{P}, \quad n=1,2, \ldots \text { and } \lim _{n \rightarrow \infty} f_{n}=f
$$

then the set

$$
A=\bigcup_{n=1}^{\infty} D\left(f_{n}\right),
$$

where $D\left(f_{n}\right)$ denotes the set of all discontinuity points of $f_{n}, n=1,2, \ldots$, is countable and the reduced function $f /(\mathbb{R} \backslash A)$ is of Baire 1 class. Consequently, there is a Baire 1 function $g: \mathbb{R} \rightarrow \mathbb{R}$ such that $f(x)=g(x)$ for all $x \in \mathbb{R} \backslash A$.

Sufficiency. Since $g$ is of Baire 1 class, there is a sequence of continuous functions $g_{n}, n=1,2, \ldots$, such that $g=\lim _{n \rightarrow \infty} g_{n}$. Let $A=\left\{a_{1}, \ldots, a_{n}, \ldots\right\}$ and for $n=1,2, \ldots$ let

$$
f_{n}(x)=\left\{\begin{array}{lcl}
f\left(a_{k}\right) & \text { for } & k \leq n \\
g_{n}(x) & \text { otherwise on } & \mathbb{R} .
\end{array}\right.
$$

Then

$$
f_{n} \in \mathcal{P} \text { for } n=1,2, \ldots \text { and } \lim _{n \rightarrow \infty} f_{n}=f .
$$

This completes the proof.

For the monotone convergence we will prove the following theorem:

Theorem 5. A function $f$ is the limit of a decreasing sequence of functions $f_{n} \in \mathcal{P}$ if and only if there are an upper semicontinuous function $g$ and $a$ countable set $A$ such that $f \leq g$ and $f(x)=g(x)$ for all points $x \in \mathbb{R} \backslash A$.

Proof. Necessity. For $n=1,2, \ldots$ and $x \in \mathbb{R}$ we define

$$
g_{n}(x)=\max \left(f_{n}(x), \lim _{t \rightarrow x-} f_{n}(t), \lim _{t \rightarrow x+} f_{n}(t)\right) .
$$

From the inequalities $f \leq f_{n+1} \leq f_{n}$ it follows that $f \leq g_{n+1} \leq g_{n}$ for $n=1,2, \ldots$. So there is a function $g$ such that $g_{n} \searrow g$ with $n \rightarrow \infty$. From the definition of $g_{n}$ and from the inclusion $f_{n} \in \mathcal{P}$ it follows that every function 
$g_{n}, n=1,2, \ldots$, is upper semicontinuous. So, the function $g$ is also upper semicontinuous and $f \leq g$.

Let

$$
A=\bigcup_{n=1}^{\infty} D\left(f_{n}\right) .
$$

The set $A$ is countable. Since all functions $f_{n}, n=1,2, \ldots$, are continuous at all points $x \in \mathbb{R} \backslash A$, we obtain

$$
g_{n}(x)=f_{n}(x) \text { for } x \in \mathbb{R} \backslash A, n=1,2, \ldots .
$$

Consequently,

$$
\{x: g(x) \neq f(x)\} \subset A
$$

and the proof of the necessity is completed.

Sufficiency. Since $g$ is upper semicontinuous, there is a decreasing sequence of continuous functions $g_{n}, n=1,2, \ldots$, such that

$$
g=\lim _{n \rightarrow \infty} g_{n} .
$$

Let

$$
A=\left\{a_{1}, a_{2}, \ldots, a_{n}, \ldots\right\}
$$

and for $n=1,2, \ldots$ let

$$
f_{n}(x)=\left\{\begin{array}{ccl}
f(x) & \text { for } & x=a_{k}, \quad k \leq n \\
g_{n}(x) & \text { otherwise on } & \mathbb{R} .
\end{array}\right.
$$

Then the functions $f_{n} \in \mathcal{P}$ for $n=1,2, \ldots$ and

$$
f_{n} \searrow f \text { with } n \rightarrow \infty .
$$

This completes the proof.

Applying the last theorem to the functions $f$ and $f_{n}, n=1,2, \ldots$, we obtain the dual version of Theorem 4 .

Theorem 6. A function $f$ is the limit of an increasing sequence of functions $f_{n} \in \mathcal{P}$ if and only if there are a lower semicontinuous function $g$ and $a$ countable set $A$ such that $f \geq g$ and

$$
\{x: f(x) \neq g(x)\} \subset A .
$$

For the monotone a.c. convergence we have the following theorems: 
Theorem 7. There are functions $f$ and $f_{n} \in \mathcal{P}, n=1,2, \ldots$, such that $f=$ a.c. $\lim _{n \rightarrow \infty} f_{n}$ and $f_{n} \nearrow f$ with $n \rightarrow \infty$ and such that for each decreasing sequence of functions $h_{n} \in \mathcal{P}, n=1,2, \ldots$, the relation $f=$ a.c. $\lim _{n \rightarrow \infty} h_{n}$ is false.

Proof. We conserve all notations from Example 3. Let $f$ be the function from Example 3. Then $f$ is the a.c. limit of the sequence of the functions $f_{n}, n=1,2, \ldots$, defined in Example 3 and belonging to $\mathcal{P}$. As an upper semicontinuous function $f$ is the limit of a decreasing sequence of continuous functions (so, belonging to $\mathcal{P}$ ).

Suppose, to the contrary that there is a decreasing sequence of functions $h_{n}, n=1,2, \ldots$, with a.c. $\lim _{n \rightarrow \infty} h_{n}=f$. Since $h_{n} \geq f$ for $n=1,2, \ldots$, for all $n, m=1,2, \ldots$ the inequality $h_{n} \geq f_{m}$ is true. There are an open interval $K$, a countable set $E$, and a positive integer $k$ such that

$$
K \cap C \neq \emptyset \text { and } h_{i}(x)=0 \text { for } x \in(K \cap C) \backslash E \text { and } i \geq k .
$$

Let $m>k$ be a positive integer with $z_{m} \in K \cap C$. In every interval $I_{m, j}$, $j=1,2, \ldots$, there is a point $u_{m, j} \in I_{m, j}$ at which $f\left(u_{m, j}\right)=\frac{1}{m}$. Consequently,

$$
h_{k}\left(u_{m, j}\right) \geq \frac{1}{m} \text { for } j=1,2, \ldots
$$

Since

$$
z_{m}=\lim _{j \rightarrow \infty} u_{m, j}
$$

and $z_{m}$ is a bilateral accumulation point of the set $K \cap(C \backslash E)$, the function $h_{k}$ has not at least one unilateral limit at $z_{m}$. So it is not in $\mathcal{P}$ and the obtained contradiction proves our theorem.

Theorem 8. Let $f$ be a function. Suppose that there are a countable set $A$, a sequence of closed sets $A_{n}$ and a sequence of functions $g_{n} \in \mathcal{P}$ with $g_{n} \geq f$ $\left(g_{n} \leq f\right), n=1,2, \ldots$, such that

$$
\mathbb{R}=\bigcup_{n=1}^{\infty} A_{n} \text { and } A_{n} \subset A_{n+1} \text { for } n=1,2, \ldots
$$

and

$$
g_{n} /\left(A_{n} \backslash A\right)=f /\left(A_{n} \backslash A\right) \text { for } n=1,2, \ldots
$$

Then there is a decreasing (increasing) sequence of functions $h_{n} \in \mathcal{P}, n=$ $1,2, \ldots$, such that $f=$ a.c. $\lim _{n \rightarrow \infty} h_{n}$. 
Proof. We will consider only the first case where $g_{n} \geq f$, since the case where $g_{n} \leq f$ is analogous.

Let $A=\left\{a_{1}, a_{2}, \ldots, a_{n}, \ldots\right\}$ and for $n=1,2, \ldots$ let

$$
h_{n}(x)=\left\{\begin{array}{ccl}
f\left(a_{i}\right) & \text { for } & i \leq n \\
\min \left(g_{1}(x), \ldots, g_{n}(x)\right) & \text { otherwise on } & \mathbb{R} .
\end{array}\right.
$$

Then the sequence of functions $h_{n} \in \mathcal{P}$ satisfies all requirements and the proof is completed.

\section{References}

[1] A. M. Bruckner, J. B. Bruckner, and B. S. Thomson, Real Analysis, Prentice-Hall International, INC, Upper Saddle River, New Jersey, 1996.

[2] A. Császár and M. Laczkovich, Discrete and equal convergence, Studia Sci. Math. Hungar. 10(1975), 463-472.

[3] R. J. O'Malley, Approximately differentiable functions. The $r$ topology, Pacific J. Math. 72(1977), 207-222.

[4] C. S. Reed, Pointwise limits of sequences of functions, Fund. Math. 67(1970), 183-193.

[5] B. S. Thomson, Real Functions, Lectures Notes in Math. 1170(1985), Springer-Verlag. 